Pacific

Journal of

Mathematics

ON THE INDEX FORMULA FOR SINGULAR SURFACES

B. Fedosov, B.-W. Schulze, and N. Tarkhanov 


\title{
ON THE INDEX FORMULA FOR SINGULAR SURFACES
}

\author{
B. Fedosov, B.-W. Schulze, and N. Tarkhanov
}

\begin{abstract}
The index formula for elliptic pseudodifferential operators on a two-dimensional manifold with conical points contains the Atiyah-Singer integral as well as two additional terms. One of the two is the 'eta' invariant defined by the conormal symbol, and the other term is explicitly expressed via the principal and subprincipal symbols of the operator at conical points. The aim of this paper is an explicit description of the contribution of a conical point for higher-order differential operators. We show that changing the origin in the complex plane reduces the entire contribution of the conical point to the shifted 'eta' invariant. In turn this latter is expressed in terms of the monodromy matrix for an ordinary differential equation defined by the conormal symbol.
\end{abstract}

\section{Introduction.}

In [FST97] we proved the following index formula for elliptic pseudodifferential operators on a two-dimensional manifold with a conical point:

$(0.1)$ ind $A=\int_{S^{*} M} \operatorname{AS}(A)-\frac{1}{2} \eta\left(A_{c}\right)+\left.\frac{i}{4 \pi^{2}} \int_{\mathbb{S}^{1} \times \mathbb{R}} \operatorname{tr} \sigma_{0}^{-1} \sigma_{\text {sub }}\right|_{\tau=-1} ^{\tau=1} d \xi d x$,

$M$ being the manifold in question whose cross-section close to the conical point is identified with the unit circle $\mathbb{S}^{1}$.

The index is evaluated for $A$ acting on weighted Sobolev spaces on $M$ as $H^{s, \gamma}\left(M, E^{0}\right) \rightarrow H^{s-m, \gamma}\left(M, E^{1}\right)$, where $E^{0}$ and $E^{1}$ are $C^{\infty}$ vector bundles over the smooth part of $M$ which behave properly when approaching the conical point.

The first term on the right-hand side of this formula is the Atiyah-Singer integral derived from the principal interior symbol $\sigma_{0}$ of $A$ and the curvature forms $\Omega^{0}$ and $\Omega^{1}$ of the bundles $E^{0}$ and $E^{1}$, respectively. We have

$$
\operatorname{AS}(A)=\frac{1}{4 \pi^{2}}\left(\frac{1}{6} \operatorname{tr}\left(\sigma_{0}^{-1} \partial \sigma_{0}\right)^{3}-\frac{1}{2} \operatorname{tr}\left(\Omega^{0} \sigma_{0}^{-1} \partial \sigma_{0}+\Omega^{1} \partial \sigma_{0} \sigma_{0}^{-1}\right)\right) .
$$

The weight exponent $\gamma$ enters only the second term on the right side of (0.1) which is known as the 'eta' invariant of the conormal symbol $A_{c}$ of $A$ 
at the conical point. More precisely,

$\eta\left(A_{c}\right)=-\frac{1}{\pi i} \overline{\operatorname{Tr}}\left(A_{c}^{-1}(\tau+i \gamma) A_{c}^{\prime}(\tau+i \gamma)-i \gamma \frac{d}{d \tau}\left(A_{c}^{-1}(\tau+i \gamma) A_{c}^{\prime}(\tau+i \gamma)\right)\right)$,

$\overline{\mathrm{Tr}}$ being a regularised trace (cf. Melrose [Mel95]).

Both these terms occur in the Atiyah-Patodi-Singer formula for the index of Dirac operators (cf. [APS75]). In contrast to this latter formula, (0.1) contains the additional third term which does not vanish even for the Cauchy-Riemann operator on the plane. This summand also depends on the conormal symbol $A_{c}(\tau)$ only because the principal symbol $\sigma_{0}$ and the so-called subprincipal symbol

$$
\sigma_{\mathrm{sub}}=\sigma_{1}+\frac{i}{2} \frac{\partial^{2} \sigma_{0}}{\partial x \partial \xi}
$$

are evaluated at the conical point. Here $\sigma_{1}$ means the homogeneous component of degree deg $\sigma_{0}-1$ of the complete symbol of $A_{c}(\tau)$.

Of course, formula (0.1) is still true for manifolds with several conical points. A slight change we have to do is that the 'eta' invariant and the additional terms should be summed up over all conical points of $M$.

The aim of this paper is an explicit description of the contribution of a conical point for elliptic differential operators. To this end we show first that by changing the origin in the complex $\tau$-plane we can make the third term to vanish reducing the whole contribution of the conical point to the shifted 'eta' invariant. The new origin $\tau_{0}$ which we refer to as the centre is the root of the linear equation

$$
\left.\int_{\mathbb{S}^{1} \times \mathbb{R}} \operatorname{tr} \sigma_{0}^{-1}\left(\frac{\partial \sigma_{0}}{\partial \tau} \tau_{0}+\sigma_{\text {sub }}\right)\right|_{\tau=-1} ^{\tau=1} d \xi d x=0 .
$$

The next goal is to express the 'eta' invariant in terms of the monodromy matrix $M(\tau)$ for an ordinary differential equation defined by the conormal symbol $A_{c}(\tau)$. We introduce a phase function

$$
\varphi(\tau)=\frac{1}{2} \log \operatorname{det}\left(M(\tau)+M^{-1}(\tau)-2\right)
$$

which is an analytic function of $\tau$ with logarithmic ramification points. Then our final index theorem reads

$$
\text { ind } A=\int_{\mathbb{S}^{*} M} \operatorname{AS}(A)+\frac{1}{2 \pi i} \Delta_{\Gamma, \tau_{0}} \varphi(\tau)
$$

where $\Delta_{\Gamma, \tau_{0}} \varphi(\tau)$ denotes the variation of the phase function along a suitable contour defined by the weight line $\Gamma$ and the centre $\tau_{0}$ (Theorem 3.1).

In some particular cases we may say more about the variation $\Delta_{\Gamma, \tau_{0}} \varphi(\tau)$. For example, if the function $f(\tau)=\operatorname{det}\left(M(\tau)+M^{-1}(\tau)-2\right)$ is even with 
respect to $\tau_{0}$, that is

$$
f\left(\tau_{0}-T\right)=f\left(\tau_{0}+T\right)
$$

then the second term in (0.3) may be calculated in terms of zeros of $f(\tau)$ and turns out to be half-integer (Theorem 3.2). Thinking over these properties we have come to a generalisation of the symmetry conditions used in [SSS97]. A detailed treatment of this symmetry in the higher-dimensional case will be given in a forthcoming paper.

Finally, we show that the above integrality of $\frac{1}{\pi i} \Delta_{\Gamma, \tau_{0}} \varphi(\tau)$ holds for any first-order elliptic system, no matter whether the symmetry condition is fulfilled or not. To this end we investigate the asymptotical behaviour of solutions and the monodromy matrix when $\Re \tau \rightarrow \pm \infty$ and $\Im \tau$ remains bounded. Although there exists vast literature on this topic, we have not found the desired facts and were forced to prove them. The proof uses the ideas of Faddeev and Takhtajan [FT87] for the non-linear Schrödinger equation.

Acknowledgments. The authors wish to express their gratitude to M. Lesch for drawing the authors' attention to the paper [BFK91] where the monodromy matrix appeared in an expression for the determinant of an elliptic differential operator on a circle.

\section{The existence of the centre.}

Recall that the neighbourhood of a conical point is treated as a cylindrical end with coordinates $t \in \mathbb{R}_{+}$and $x \in \mathbb{R} \bmod (2 \pi)$. Since any complex vector bundle over a circle is trivial, we may assume that $E^{0} \cong E^{1} \cong \mathbb{C}^{r}$ over the cylindrical end and, for given trivialisations, the connection one-forms $\Gamma^{0}$, $\Gamma^{1}$ are equal to 0 .

The conormal symbol of an $m$ th order differential operator has the form

$$
A_{c}(\tau)=a_{m}(x) \frac{\partial^{m}}{\partial x^{m}}+a_{m-1}(x, \tau) \frac{\partial^{m-1}}{\partial x^{m-1}}+\ldots+a_{0}(x, \tau) .
$$

So, it is an ordinary differential operator on a circle whose coefficients

$$
a_{k}(x, \tau)=\sum_{l=0}^{m-k} a_{k, l}(x) \tau^{l}
$$

are polynomials in $\tau$ of degree $m-k$. Thus, the principal symbol of the operator $A$ restricted to the boundary is

$$
\sigma_{0}(A)=\sum_{k=0}^{m} a_{m-k, k}(x) \tau^{k}(i \xi)^{m-k}
$$


and for the lower-order term we have

$$
\sigma_{1}(A)=\sum_{k=0}^{m-1} a_{m-1-k, k}(x) \tau^{k}(i \xi)^{m-1-k} .
$$

The interior ellipticity means that $\sigma_{0}(A)$ is an invertible matrix for any real $(\xi, \tau) \neq(0,0)$; in particular, the coefficient $a_{m}(x)$ in (1.1) is an invertible matrix-valued function on a circle. Without loss of generality we assume that $a_{m}(x) \equiv 1$, otherwise we change the frame in $E^{1}$ using $a_{m}$ as a transition matrix.

Replacing $\tau$ by $\tau+\tau_{0}$ in (1.1), we see that the shift by $\tau_{0}$ in the complex $\tau$-plane does not change the principal symbol $\sigma_{0}$, while for $\sigma_{1}$ we have a new expression

$$
\widetilde{\sigma_{1}}=\sigma_{1}+\frac{\partial \sigma_{0}}{\partial \tau} \tau_{0}
$$

The subprincipal symbol $\sigma_{\text {sub }}$ obeys the same rule

$$
\widetilde{\sigma_{\text {sub }}}=\sigma_{\text {sub }}+\frac{\partial \sigma_{0}}{\partial \tau} \tau_{0}
$$

Thus, after shifting we obtain a new additional term in (0.1) proportional to the left-hand side of (0.2). The following theorem guaranties a unique solvability of the linear equation (0.2).

Theorem 1.1. For any elliptic differential operator $A$,

$$
\left.\int_{\mathbb{S}^{1} \times \mathbb{R}} \operatorname{tr} \sigma_{0}^{-1} \frac{\partial \sigma_{0}}{\partial \tau}\right|_{\tau=-1} ^{\tau=1} d x d \xi \neq 0 .
$$

Proof. Denoting det $\sigma_{0}$ by $f(x, \tau, \xi)$, we have

$$
\operatorname{tr} \sigma_{0}^{-1} \frac{\partial \sigma_{0}}{\partial \tau}=f^{-1} \frac{\partial f}{\partial \tau}
$$

From ellipticity we deduce that the roots of the polynomial $f=f(\tau, \xi)$ for fixed real $\tau$ form two disjoint sets corresponding to the upper and lower halfplanes. The integrand in (1.2) is a rational function in $\xi$ decaying as $O\left(|\xi|^{-2}\right)$ when $\xi \rightarrow \infty$. Thus, integrating over $\xi$, we may replace the real axis by a closed contour $c_{ \pm}$consisting of a large semicircle in the upper (lower) halfplane and its diameter and surrounding all the poles in the corresponding half-plane.

By the Euler theorem for homogeneous functions,

$$
f^{-1} \frac{\partial f}{\partial \tau}=\frac{1}{\tau}\left(m r-\xi f^{-1} \frac{\partial f}{\partial \xi}\right)
$$

so that

$$
\left.f^{-1} \frac{\partial f}{\partial \tau}\right|_{\tau=-1} ^{\tau=1}=2 m r-\left.\xi f^{-1} \frac{\partial f}{\partial \xi}\right|_{\tau=1}-\left.\xi f^{-1} \frac{\partial f}{\partial \xi}\right|_{\tau=-1}
$$


and the residue theorem yields

$$
\left.\int_{\mathbb{R}} f^{-1} \frac{\partial f}{\partial \tau}\right|_{\tau=-1} ^{\tau=1} d \xi=-2 \pi i\left(\sum \xi_{k}^{+}+\sum \xi_{k}^{-}\right)
$$

where $\xi_{k}^{ \pm}$are the roots of the equation $f( \pm 1, \xi)=0$ in the upper half-plane. Since $f( \pm 1, \xi)=0$ is equivalent to $f(1, \pm \xi)=0$, we see that at least one set $\xi^{+}$or $\xi^{-}$is not empty. Thus,

$$
\Im\left(\sum \xi_{k}^{+}+\sum \xi_{k}^{-}\right)>0
$$

proving the theorem.

Remark 1.2. Our proof uses essentially the fact that $f(\tau, \xi)$ is a homogeneous polynomial. Clearly, for rational homogeneous functions $f(\tau, \xi)$ having no zeros and poles on the real axis $\Im \xi=0$ the theorem is not true.

\section{The Green function and the monodromy matrix.}

The operator

$$
\frac{d}{d \tau}\left(A_{c}^{-1} \frac{d}{d \tau} A_{c}(\tau)\right)
$$

is a pseudodifferential operator of order -2 on the circle, thus it belongs to the trace class. Its trace may be explicitly calculated in terms of the so-called monodromy matrix.

Consider the ordinary differential equation

$$
A_{c}(\tau) u=u^{(m)}(x)+a_{m-1}(x, \tau) u^{(m-1)}(x)+\ldots+a_{0}(x, \tau) u(x)=0 .
$$

Its solutions form a linear space of dimension $m r$. Since the coefficients are $2 \pi$-periodic functions, the shift $u(x) \mapsto u(x+2 \pi)$ defines a linear transformation $M$ of the space of solutions called the monodromy.

Theorem 2.1. The monodromy transformations $M(\tau), M^{-1}(\tau)$ are entire functions in $\tau$, and

$$
\operatorname{Tr} \frac{d}{d \tau}\left(A_{c}^{-1} \frac{d}{d \tau} A_{c}(\tau)\right)=\frac{1}{2} \frac{d^{2}}{d \tau^{2}} \log \operatorname{det}\left(M(\tau)+M^{-1}(\tau)-2\right) .
$$

Proof. Any solution $u(x)$ is uniquely defined by the vector of its Cauchy data

$$
\vec{u}(x)=\left(\begin{array}{c}
u(x) \\
u^{\prime}(x) \\
\cdots \\
u^{(m-1)}(x)
\end{array}\right)
$$

at some point $x_{0}$. The monodromy carries the vector $\vec{u}\left(x_{0}\right)$ to $\vec{u}\left(x_{0}+2 \pi\right)$ and we may calculate the monodromy matrix as follows. Consider the Wronsky 
matrix $U\left(x, x_{0}, \tau\right)$ consisting of linearly independent vector-valued functions (2.2) normalised by the initial condition

$$
\left.U\right|_{x=x_{0}}=U\left(x_{0}, x_{0}, \tau\right)=1 .
$$

Then,

$$
\begin{aligned}
M(\tau) & =U\left(x_{0}+2 \pi, x_{0}, \tau\right), \\
M^{-1}(\tau) & =U\left(x_{0}-2 \pi, x_{0}, \tau\right) .
\end{aligned}
$$

The Wronsky matrix satisfies a first-order differential equation

$$
\mathcal{A}(\tau) U=0
$$

where $\mathcal{A}(\tau)$ is given by the block matrix

$$
\mathcal{A}(\tau)=\left(\begin{array}{cclc}
d / d x & -1 & \ldots & 0 \\
0 & d / d x & \ldots & 0 \\
\ldots & \ldots & \ldots & \ldots \\
0 & 0 & \ldots & -1 \\
a_{0}(x, \tau) & a_{1}(x, \tau) & \ldots & a_{m-1}(x, \tau)+d / d x
\end{array}\right)
$$

Since the coefficients are polynomials in $\tau$, the solution $U\left(x, x_{0}, \tau\right)$ is a holomorphic function in $\tau \in \mathbb{C}$ and so are $M(\tau)$ and $M^{-1}(\tau)$. We will express the operator $A_{c}^{\prime}(\tau) A_{c}^{-1}(\tau)$ through the first order operator $(2.5)$.

Introduce the notion $\sum \mathcal{B}$ for the sum of diagonal blocks of a block matrix $\mathcal{B}$.

Lemma 2.2. The following equality holds

$$
A_{c}^{\prime}(\tau) A_{c}^{-1}(\tau)=\sum \mathcal{A}^{\prime}(\tau) \mathcal{A}^{-1}(\tau) .
$$

Proof. To find $\mathcal{A}^{-1}(\tau)$, write the equation $\mathcal{A} \vec{u}(\tau)=\vec{v}$ for $\vec{u}$ in components

$$
\begin{aligned}
& \frac{d u_{0}}{d x}-u_{1}=v_{0} \\
& \frac{d u_{1}}{d x}-u_{2}=v_{1} \\
& \frac{d u_{m-1}}{d x}+a_{m-1} u_{m-1}+\ldots+a_{0} u_{0}=v_{m-1} .
\end{aligned}
$$

Eliminating

$$
\begin{aligned}
u_{1} & =\frac{d u_{0}}{d x}-v_{0}, \\
u_{2} & =\frac{d}{d x}\left(\frac{d u_{0}}{d x}-v_{0}\right)-v_{1}
\end{aligned}
$$

and so on, we obtain an equation for $u_{0}$ of the form

$$
(d / d x)^{m} u_{0}+a_{m-1}(d / d x)^{m-1} u_{0}+\ldots+a_{0} u_{0}=w
$$


where $w$ is a known function, namely a linear combination of $v_{0}, v_{1}, \ldots, v_{m-1}$ and their derivatives. Thus, $u_{0}=A_{c}^{-1}(\tau) w$, and moving backward we find successively $u_{1}, u_{2}, \ldots, u_{m-1}$. The most simple expression we have in the case when $v_{0}=v_{1}=\ldots=v_{m-2}=0$. Then

$$
\begin{aligned}
u_{0} & =A_{c}^{-1}(\tau) v_{m-1}, \\
u_{1} & =(d / d x) A_{c}^{-1}(\tau) v_{m-1}, \\
\cdots & \cdots \cdots \\
u_{m-1} & =(d / d x)^{m-1} A_{c}^{-1}(\tau) v_{m-1} .
\end{aligned}
$$

It follows that $\mathcal{A}^{-1}$ exists exactly when $A_{c}^{-1}$ does and

$$
\mathcal{A}^{-1}(\tau)=\left(\begin{array}{cccc}
* & \ldots & * & A_{c}^{-1}(\tau) \\
* & \ldots & * & (d / d x) A_{c}^{-1}(\tau) \\
\ldots & \ldots & \ldots & \ldots \\
* & \ldots & * & (d / d x)^{m-1} A_{c}^{-1}(\tau)
\end{array}\right)
$$

where $*$ means any expression whose explicit form is irrelevent. Next,

$$
\mathcal{A}^{\prime}(\tau)=\left(\begin{array}{cccc}
0 & 0 & \ldots & 0 \\
\ldots & \ldots & \ldots & \ldots \\
0 & 0 & \ldots & 0 \\
a_{0}^{\prime} & a_{1}^{\prime} & \ldots & a_{m-1}^{\prime}
\end{array}\right)
$$

the prime meaning the derivation in $\tau$, so that

$$
\mathcal{A}^{\prime}(\tau) \mathcal{A}^{-1}(\tau)=\left(\begin{array}{cccc}
0 & 0 & \ldots & 0 \\
\ldots & \ldots & \ldots & \ldots \\
0 & 0 & \ldots & 0 \\
* & * & \ldots & A_{c}^{\prime}(\tau) A_{c}^{-1}(\tau)
\end{array}\right)
$$

proving the lemma.

Now we find $\mathcal{A}^{-1}(\tau)$ in another way. Let $x$ vary in the closed interval $[0,2 \pi]$ and let $U(x, \tau)$ be the Wronsky matrix satisfying $(2.3)$ at $x_{0}=0$. The operator $\mathcal{A}^{-1}(\tau)$, when considered on periodic functions on $[0,2 \pi]$, is an integral operator whose kernel $G(x, y, \tau)$ (the Green function) is a periodic solution of the equation

$$
\mathcal{A}(\tau) G(x, y, \tau)=\delta(x-y) .
$$

We treat $x$ as an argument while $y \in[0,2 \pi]$ is considered as a parameter, $\delta$ being the Dirac $\delta$-function. This equation means that $G$ satisfies the homogeneous equation on $[0, y)$ and $(y, 2 \pi]$, whence

$$
\begin{array}{lll}
G(x, y, \tau)=U(x, \tau) C_{-}, & \text {for } \quad x \in[0, y), \\
G(x, y, \tau)=U(x, \tau) C_{+}, & \text {for } \quad x \in(y, 2 \pi],
\end{array}
$$


the matrices $C_{ \pm}$being independent of $x$. To produce the $\delta$-function, these matrices should satisfy the relation

$$
C_{+}-C_{-}=U^{-1}(y, \tau)
$$

while periodicity yields

$$
\begin{aligned}
C_{-} & =U(2 \pi, \tau) C_{+} \\
& =M(\tau) C_{+} .
\end{aligned}
$$

Solving this system, we get a usual expression for the Green function, namely

$$
G(x, y, \tau)= \begin{cases}U(x, \tau)(1-M(\tau))^{-1} U^{-1}(y, \tau), & x \in[0, y), \\ U(x, \tau) M(\tau)(1-M(\tau))^{-1} U^{-1}(y, \tau), & x \in(y, 2 \pi]\end{cases}
$$

or equivalently

$$
\begin{aligned}
G(x, y, \tau)= & \frac{1}{2} U(x, \tau)(1+M(\tau))(1-M(\tau))^{-1} U^{-1}(y, \tau) \\
& +\frac{1}{2} \operatorname{sgn}(x-y) U(x, \tau) U^{-1}(y, \tau) .
\end{aligned}
$$

Using Lemma 2.2, we conclude that the operator $\left(A_{c}^{\prime}(\tau) A_{c}^{-1}(\tau)\right)^{\prime}$ has the kernel

$$
\begin{aligned}
\frac{1}{2} \sum & \frac{d}{d \tau}\left(\mathcal{A}^{\prime}(\tau) U(x, \tau)(1+M(\tau))(1-M(\tau))^{-1} U^{-1}(y, \tau)\right) \\
+ & \frac{1}{2} \operatorname{sgn}(x-y) \sum \frac{d}{d \tau}\left(\mathcal{A}^{\prime}(\tau) U(x, \tau) U^{-1}(y, \tau)\right)
\end{aligned}
$$

The second term vanishes at $x=y$ because from (2.6)

$$
\begin{aligned}
\sum \frac{d}{d \tau} \mathcal{A}^{\prime}(\tau) & =a_{m-1}^{\prime \prime}(\tau) \\
& =0
\end{aligned}
$$

since $a_{m-1}$ is a linear function in $\tau$. To calculate the trace of $\left(A_{c}^{\prime}(\tau) A_{c}^{-1}(\tau)\right)^{\prime}$ (which belongs to the trace class), we put $x=y$ in (2.7), take the matrix trace and integrate over $[0,2 \pi]$. The second term in (2.7) may be dropped and we obtain

$$
\begin{aligned}
& \operatorname{Tr}\left(A_{c}^{\prime}(\tau) A_{c}^{-1}(\tau)\right)^{\prime} \\
& =\int_{0}^{2 \pi} \operatorname{tr} \frac{\partial G}{\partial \tau}(x, x, \tau) d x \\
& =\frac{1}{2} \frac{d}{d \tau} \operatorname{tr}(1+M(\tau))(1-M(\tau))^{-1} \int_{0}^{2 \pi} U^{-1}(x, \tau) \mathcal{A}^{\prime}(x, \tau) U(x, \tau) d x .
\end{aligned}
$$

To complete the proof of Theorem 2.1, we need the following lemma. 
Lemma 2.3. We have

$$
M^{-1}(\tau) M^{\prime}(\tau)=-\int_{0}^{2 \pi} U^{-1}(x, \tau) \mathcal{A}^{\prime}(x, \tau) U(x, \tau) d \tau .
$$

Proof. Differentiating (2.4) in $\tau$, we obtain

$$
\mathcal{A}(\tau) U^{\prime}+\mathcal{A}^{\prime}(\tau) U=0
$$

with an initial condition

$$
\left.U^{\prime}(x, \tau)\right|_{x=0}=0,
$$

where the prime means derivation in $\tau$. To find $U^{\prime}$, we apply a variation of constants to (2.8) looking for $U^{\prime}$ in the form $U V$. Then (2.8) yields

$$
U \frac{d V}{d x}+\mathcal{A}^{\prime}(\tau) U=0
$$

so that

$$
V(x)=-\int_{0}^{x} U^{-1}(y, \tau) \mathcal{A}^{\prime}(y, \tau) U(y, \tau) d y
$$

and

$$
U^{\prime}(x, \tau)=U(x, \tau) \int_{0}^{x} U^{-1}(y, \tau) \mathcal{A}^{\prime}(y, \tau) U(y, \tau) d y .
$$

Taking $x=2 \pi$ yields the desired identity.

Now

$$
\begin{aligned}
\operatorname{Tr}\left(A_{c}^{\prime}(\tau) A_{c}^{-1}(\tau)\right)^{\prime} & =-\frac{1}{2} \frac{d}{d \tau} \operatorname{tr}(1+M)(1-M)^{-1} M^{-1} M^{\prime} \\
& =\frac{d}{d \tau} \operatorname{tr}(M-1)^{-1} M^{\prime}-\frac{1}{2} \frac{d}{d \tau} \operatorname{tr} M^{-1} M^{\prime} \\
& =\frac{1}{2} \frac{d^{2}}{d \tau^{2}} \log \operatorname{det}(M-1)^{2} M^{-1}
\end{aligned}
$$

which is precisely $(2.1)$.

\section{The index formula.}

Combining the results of Sections 1 and 2, we obtain a simple interpretation of the boundary terms in the index formula (0.1). We also introduce a symmetry condition generalising that of [SSS97]. It allows one to simplify further the boundary term reducing it to the number of poles of $A_{c}^{-1}$ in a strip.

Consider two horizontal lines $\Gamma, \Gamma_{0}$ in the complex $\tau$-plane, $\Gamma_{0}$ passing through the centre $\tau_{0}$. In the strip between these lines the operator $A_{c}^{-1}(\tau)$ has a finite number of poles. In particular, for $|\Re \tau|>T_{0} \gg 1$ there are no poles at all. Consider a contour starting at the point $\tau_{0}-T$ with $T>0$ 
large enough, so that $\left|\Re\left(\tau_{0}-T\right)\right|>T_{0}$, then going along $\Gamma$ in the region where $|\Re \tau|<T_{0}$, and terminating at the point $\tau_{0}+T$ (see Fig. 1).

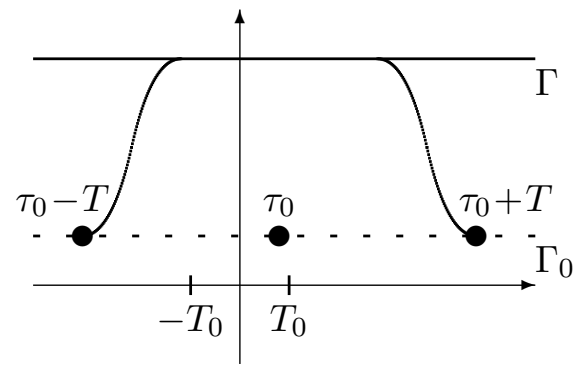

Figure 1. Variation of $\varphi(t)$ along $\Gamma$.

The function

$$
\varphi(\tau)=\frac{1}{2} \log \operatorname{det}\left(M+M^{-1}-2\right)
$$

is analytic with ramification points at zeros of

$$
\begin{aligned}
f(\tau) & =e^{\varphi(\tau)} \\
& =\operatorname{det}^{1 / 2}\left(M+M^{-1}-2\right) \\
& =\frac{\operatorname{det}(M-1)}{\operatorname{det}^{1 / 2} M} .
\end{aligned}
$$

Clearly, the zeros of $\operatorname{det}(M(\tau)-1)$ are the poles of $A_{c}^{-1}(\tau)$. Denote by $\varphi\left(\tau_{0}+T\right)-\varphi\left(\tau_{0}-T\right)$ the variation of $\varphi(\tau)$ along the contour described above and set

$$
\Delta_{\Gamma, \tau_{0}} \varphi(\tau)=\lim _{T \rightarrow \infty}\left(\varphi\left(\tau_{0}+T\right)-\varphi\left(\tau_{0}-T\right)\right) .
$$

With this notation we have the following theorem.

Theorem 3.1. Let $\tau_{0}$ be the centre and $M(\tau)$ be the monodromy matrix of the ordinary differential operator $A_{c}(\tau)$ on the circle. Then

$$
\text { ind } A=\int_{\mathbb{S}^{*} M} \operatorname{AS}(A)+\frac{1}{2 \pi i} \Delta_{\Gamma, \tau_{0}} \varphi(\tau) .
$$

Proof. Let us consider the isomorphisms of the bundles $E^{0}, E^{1}$ consisting in multiplication by $\exp \left(i \tau_{0} t\right)$. The local expressions for the operator $A=$ $A_{c}(-i \partial / \partial t)$ in cylindrical charts change to

$$
e^{-i \tau_{0} t} A e^{i \tau_{0} t}=A_{c}\left(-i \frac{\partial}{\partial t}+\tau_{0}\right)
$$

So, its conormal symbol changes to $A_{c}\left(\tau+\tau_{0}\right)$. 
If $\tau_{0}$ is the centre, then according to Section 1 the boundary term consists of

$$
-\frac{1}{2} \eta\left(A_{c}\left(\tau+\tau_{0}\right)\right)=\frac{1}{2 \pi i} \overline{\operatorname{Tr}} A_{c}^{-1}\left(\tau+\tau_{0}\right) A_{c}^{\prime}\left(\tau+\tau_{0}\right) .
$$

It is sufficient to compute (3.3) for $\tau_{0}=0$. Consider

$$
Q(\tau)=\overline{\operatorname{Tr}} \frac{\partial}{\partial \tau}\left(A_{c}^{-1}(\tau) A_{c}^{\prime}(\tau)-i \gamma \frac{\partial}{\partial \tau} A_{c}^{-1}(\tau) A_{c}^{\prime}(\tau)\right) .
$$

By Theorem 2.1, this quantity is equal to

$$
\frac{\partial^{2}}{\partial \tau^{2}}\left(\varphi(\tau)-i \gamma \frac{\partial}{\partial \tau} \varphi(\tau)\right)
$$

According to the definition of $\overline{\operatorname{Tr}}$ (see Melrose [Mel95]) and $\eta\left(A_{c}\right)$, we obtain

$$
-\frac{1}{2} \eta\left(A_{c}\right)=\lim _{T \rightarrow \infty} \int_{-T}^{T} d \tau_{1} \int_{0}^{\tau_{1}} Q(\tau+i \gamma) d \tau,
$$

the right-hand side being understood as a constant term in the asymptotic expansion when $T \rightarrow \infty$. Thus,

$$
-\frac{1}{2} \eta\left(A_{c}\right)=\left.\frac{1}{2 \pi i} \lim _{T \rightarrow \infty}\left(\varphi(\tau)-i \gamma \frac{\partial}{\partial \tau} \varphi(\tau)\right)\right|_{\tau=-T+i \gamma} ^{\tau=T+i \gamma}
$$

and the variation of $\varphi(\tau)$ is taken along the weight line $\Gamma$ (for $(\partial / \partial \tau) \varphi(\tau)$, the variation does not depend on the path). In the region $|\Re \tau|>T_{0}$ where $\varphi(\tau)$ is holomorphic in the strip between $\Gamma$ and the real axis, we may use the Taylor formula, thus obtaining

$$
\varphi(\tau)-i \gamma \frac{\partial}{\partial \tau} \varphi(\tau)=\varphi(\tau-i \gamma)+R_{2}(\tau, \gamma)
$$

where $R_{2}(\tau, \gamma)$ is a remainder term which tends to 0 for $\Re \tau \rightarrow \pm \infty$ and $|\Im \tau| \leq C$. Hence it follows that

$$
-\frac{1}{2} \eta\left(A_{c}\right)=\frac{1}{2 \pi i} \lim _{T \rightarrow \infty}(\varphi(T)-\varphi(-T)),
$$

the variation is taken along the contour in Fig. 1 with $\tau_{0}=0$. This completes the proof.

There are important particular cases when the variation (3.1) can be calculated by the residue theorem.

Theorem 3.2. Let $f^{2}(\tau)=\operatorname{det}\left(M(\tau)+M^{-1}(\tau)-2\right)$ be an even function with respect to the centre $\tau_{0}$, that is

$$
f^{2}\left(\tau_{0}+T\right)=f^{2}\left(\tau_{0}-T\right)
$$


for any $T$. Then

$$
\text { ind } A=\int_{\mathbb{S}^{*} M} \operatorname{AS}(A)+\left(p+\frac{1}{2} q\right) \operatorname{sgn}\left(\Im \tau_{0}-\gamma\right)
$$

where $p$ is the number of zeros of $f(\tau)$ (counted along with their multiplicities) in the strip between $\Gamma$ and $\Gamma_{0}$, and $q$ is the number of zeros on the line $\Gamma_{0}$.

Proof. To be specific, let $\Im \tau_{0}<\gamma$. Consider a closed contour $l=l_{1} \cup l_{2}$ where $l_{1}$ is the contour on Fig. 1 and $l_{2}$ goes along the line $\Gamma_{0}$ bypassing the zeros lying on $\Gamma_{0}$ along small semicircles (see Fig. 2).

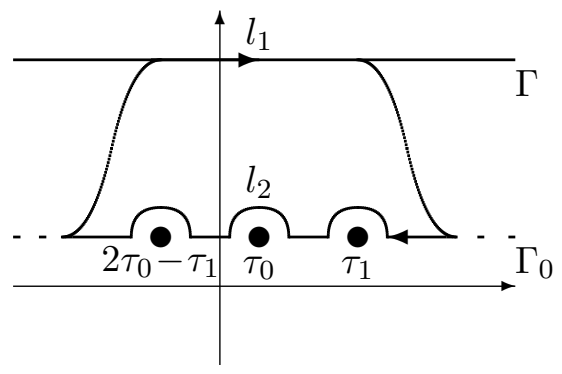

Figure 2. The contour $l=l_{1} \cup l_{2}$.

Clearly,

$$
\begin{aligned}
\frac{1}{2 \pi i} \Delta_{\Gamma, \tau_{0}} \varphi(\tau) & =\frac{1}{2 \pi i} \Delta_{l_{1}} \varphi(\tau) \\
& =-p-\frac{1}{2 \pi i} \Delta_{l_{2}} \varphi(\tau) .
\end{aligned}
$$

We next observe that the variation of $\varphi(\tau)$ along $l_{2}$ is equal to the sum of variations along all the semicircles. Indeed, the variations along the segments of $\Gamma_{0}$ cancel because of (3.4). When the radii of the semicircles tend to 0 , the variations along them tend to $\pi i$ times the number $q$ of zeros on $\Gamma_{0}$ counted together with their multiplicities. This is the desired conclusion.

Since the result is very simple, it is desirable to have simple sufficient conditions for (3.4) to be fulfilled. One of these is the symmetry condition of [SSS97] for the conormal symbol: there exist isomorphisms $v_{0}(x)$ and $v_{1}(x)$ of the bundles $E^{0}$ and $E^{1}$, such that

$$
A_{c}\left(\tau_{0}-T\right)=v_{1}(x) A_{c}\left(\tau_{0}+T\right) v_{0}(x)
$$

for each real $T$. Roughly speaking (3.6) means that the symmetry transformation $\tau \mapsto 2 \tau_{0}-\tau$ acts on $A_{c}(\tau)$ by an automorphism of the algebra of differential operators on $\mathbb{S}^{1}$ induced by isomorphisms of the bundles $E^{0}, E^{1}$. 
We introduce more general symmetry conditions including automorphisms generated by changes of variables.

Definition 3.3. The conormal symbol $A_{c}(\tau)$ is called symmetric (with respect to the centre $\tau_{0}$ ) if there exist a diffeomorphism $g: \mathbb{S}^{1} \rightarrow \mathbb{S}^{1}$ and bundle isomorphisms

$$
\begin{array}{ll}
v_{0}: & g^{*} E^{0} \rightarrow E^{0} \\
v_{1}: & g^{*} E^{1} \rightarrow E^{1}
\end{array}
$$

such that

$$
A_{c}\left(\tau_{0}-T\right)=\left(g^{-1}\right)^{*} v_{1}^{-1} A_{c}\left(\tau_{0}+T\right) v_{0} g^{*} .
$$

The definition gains in interest if we realise that differential operators with symmetric conormal symbols meet the condition of Theorem 3.2.

Proposition 3.4. For symmetric conormal symbols (3.4) holds.

Proof. A diffeomorphism $g: \mathbb{S}^{1} \rightarrow \mathbb{S}^{1}$ is defined by a monotone function $g(x)$, $x \in \mathbb{R}^{1}$, such that

$$
g(x+2 \pi)=g(x) \pm 2 \pi
$$

where the sign ' + ' means that $g$ preserves the orientation while ' - ' corresponds to diffeomorphisms reversing the orientation. If $U\left(x, \tau_{0}-T\right)$ is the Wronsky matrix for $A_{c}\left(\tau_{0}-T\right)$, then by (3.7) we have

$$
U\left(x, \tau_{0}+T\right)=v_{0}(x) U\left(g(x), \tau_{0}-T\right) v_{0}^{-1}(x) .
$$

Taking $x=2 \pi$, we obtain

$$
M\left(\tau_{0}+T\right)=v_{0}(0)\left(M^{ \pm 1}\left(\tau_{0}-T\right)\right) v_{0}^{-1}(0) .
$$

Thus, in the case of orientation-preserving diffeomorphisms $g$ (in particular, under the symmetry condition (3.6)) we have

$$
M\left(\tau_{0}+T\right)=v_{0}(0) M\left(\tau_{0}-T\right) v_{0}^{-1}(0),
$$

while an orientation-reversing diffeomorphism $g$ yields

$$
M\left(\tau_{0}+T\right)=v_{0}(0) M^{-1}\left(\tau_{0}-T\right) v_{0}^{-1}(0) .
$$

Both these properties imply (3.4) and thus (3.5).

Consider some examples illustrating Proposition 3.4.

Example 3.5. Let $\tau_{0}=0 ; g: x \mapsto-x$ and $v_{0}=1, v_{1}=(-1)^{m}$. Then (3.7) written for

$$
A_{c}(\tau)=\sum_{k+l \leq m} a_{k, l}(x) \tau^{k} \frac{\partial^{l}}{\partial x^{l}}
$$

just amounts to the fact that

$$
a_{k, l}(-x)=(-1)^{m+k+l} a_{k, l}(x) .
$$


In other words, the coefficients are even matrix functions if $k+l$ and $m$ have the same parity, and odd functions otherwise. In particular, constant coefficients will do, provided that $a_{k, l}=0$ for $k+l \not \equiv m(\bmod 2)$.

Example 3.6. For a first-order scalar differential operator

$$
A_{c}(\tau)=\frac{d}{d x}-a(x) \tau-b(x)
$$

Proposition 3.4 always holds. Indeed, the monodromy is given by a scalar factor

$$
M(\tau)=\exp \left(\int_{0}^{2 \pi}(a(x) \tau+b(x)) d x\right)
$$

and the centre $\tau_{0}$ is the root of Equation (0.2) which in our case reduces to

$$
\int_{0}^{2 \pi}\left(a(x) \tau_{0}+b(x)\right) d x=0 .
$$

Clearly, $M\left(\tau_{0}-T\right)=M^{-1}\left(\tau_{0}+T\right)$.

A particular case of this example is the Cauchy-Riemann operator on a Riemann surface with conical points.

Remark 3.7. It is interesting that the index formula in the form (3.5) under symmetry condition (3.7) is valid in the general setting of pseudodifferential operators on a higher-dimensional manifold with conical singularities. The proof using the ideas of [SSS97] and the machinery of [FST97] will be given in a forthcoming paper.

\section{First-order operators.}

Consider in more detail the case of a first-order matrix-valued operator

$$
A_{c}(\tau)=\frac{d}{d x}-A(x) \tau-B(x)
$$

We will show that, similarly to Example 3.6, the centre is completely determined by the monodromy matrix, or rather by its asymptotic behaviour when $\Re \tau \rightarrow \pm \infty$ while $\Im \tau$ remains bounded. The asymptotics implies that the boundary contribution in the index formula (3.2) is half-integer provided the frames in $E^{0}, E^{1}$ are chosen in an appropriate way. Consequently, the Atiyah-Singer term also has a half-integer value. The interpretation in terms of zeros as in (3.5) fails in general.

We begin with a choice of frames in $E^{0}$ and $E^{1}$. By the interior ellipticity, the spectrum of $A(x)$ at any $x \in \mathbb{S}^{1}$ does not intersect the imaginary axis, 
so it consists of two disjoint parts in the right and left half-planes. The corresponding spectral projectors are given by the Cauchy integrals

$$
P_{ \pm}(x)=\frac{1}{2 \pi i} \int_{c_{ \pm}}(\xi+i A(x))^{-1} d \xi
$$

where the contours $c_{ \pm}$surround the spectrum in the corresponding halfplanes. These projectors depend smoothly on $x$ defining a splitting of the trivial bundle $\mathbb{C}^{r} \cong E^{0} \cong E^{1}$ into a direct sum of two subbundles. Like any complex bundle over a circle, these subbundles are trivial. It follows that we may choose a frame in $\mathbb{C}^{r}$ with a transition matrix $C(x)$, so that

$$
\begin{aligned}
& P_{+}(x)=C(x)\left(\begin{array}{ll}
1 & 0 \\
0 & 0
\end{array}\right) C^{-1}(x), \\
& P_{-}(x)=C(x)\left(\begin{array}{ll}
0 & 0 \\
0 & 1
\end{array}\right) C^{-1}(x)
\end{aligned}
$$

and

$$
A_{1}^{-1}(x) A_{2}(x)=i C(x)\left(\begin{array}{cc}
a_{+}(x) & 0 \\
0 & a_{-}(x)
\end{array}\right) C^{-1}(x),
$$

where $a_{ \pm}(x)$ are $\left(r_{ \pm} \times r_{ \pm}\right)$-matrices having the spectra in the right (left) halfplane. Passing to new frames in $E^{0}, E^{1}$ with the same transition matrix $C(x)$, we reduce the matrix $A(x)$ to a block-diagonal form

$$
A(x)=\left(\begin{array}{cc}
a_{+}(x) & 0 \\
0 & a_{-}(x)
\end{array}\right) .
$$

Here the matrices $a_{ \pm}$have their spectra in the right (left) half-planes. The matrix $B(x)$ changes to

$$
C^{-1} B C+C^{-1} \frac{d C}{d x}
$$

and may be written in a block form

$$
B(x)=\left(\begin{array}{ll}
b_{11}(x) & b_{12}(x) \\
b_{21}(x) & b_{22}(x)
\end{array}\right)
$$

corresponding to (4.3). Thus, we have reduced the conormal symbol $A_{c}(\tau)$ to the canonical form (4.1), (4.3), (4.4) with a block-diagonal matrix $A(x)$.

Lemma 4.1. The centre $\tau_{0}$ is the root of the equation

$$
\int_{0}^{2 \pi}\left(\operatorname{tr}\left(a_{+}(x) \tau+b_{11}(x)\right)-\operatorname{tr}\left(a_{-}(x) \tau+b_{22}(x)\right)\right) d x=0 .
$$

Proof. We have

$$
\begin{aligned}
\sigma_{0} & =i \xi-A(x) \tau \\
\sigma_{\text {sub }} & =-B(x)
\end{aligned}
$$


so Equation (0.2) reduces to

$$
\operatorname{tr} \int_{0}^{2 \pi} d x \int_{-\infty}^{\infty}\left((\xi+i A(x))^{-1}-(\xi-i A(x))^{-1}\right)\left(A(x) \tau_{0}+B(x)\right) d \xi=0 .
$$

Integrating over $\xi$ and using (4.2), we obtain

$$
\operatorname{tr} \int_{0}^{2 \pi}\left(\begin{array}{ll}
1 & 0 \\
0 & 1
\end{array}\right)\left(A(x) \tau_{0}+B(x)\right) d x=0,
$$

which is precisely (4.5).

\section{Lyapunov estimates.}

In this section we consider the so-called stable case when the part $a_{+}(x)$ in (4.3) is absent. So, all the eigenvalues of $A(x)=a_{-}(x)$ have negative real parts. Such matrices will be called stable. The Wronsky matrix as a function of $x$ is a solution of the Cauchy problem

$$
\begin{aligned}
\frac{d U}{d x} & =(A(x) \tau+B(x)) U, \\
\left.U\right|_{x=y} & =1 .
\end{aligned}
$$

The following theorem gives an estimate for the fundamental solution $U(x, y, \tau)=U(x, \tau) U^{-1}(y, \tau)$.

Theorem 5.1. Let $A(x)$ be a stable matrix. Then there exist constants $C, d>0$ such that, for $\tau \gg 1$,

$$
\|U(x, y, \tau)\| \leq C \exp (-d(x-y) \tau),
$$

provided $x \geq y$, where $\|\cdot\|$ means any matrix norm.

Remark 5.2. In the case of constant coefficients $A$ and $B$, estimate (5.1) is obvious, because the solutions can be expressed in terms of exponential functions. For variable coefficients it is not, however, so obvious (recall stable and unstable zones for the Schrödinger equation).

The following necessary and sufficient condition of stability is due to Lyapunov (see e.g. [Gan86]).

Lemma 5.3. A complex matrix $A$ is stable if and only if there exists a Hermitian positive definite matrix $X$ such that

$$
A^{*} X+X A=-1 \text {. }
$$

Proof. If $A$ is stable, so is $A^{*}$. Hence both $\exp A t$ and $\exp A^{*} t$ are exponentially decaying as $t \rightarrow+\infty$. The matrix $X$ may be defined by an explicit expression, namely

$$
X=\int_{0}^{\infty} \exp \left(A^{*} t\right) \exp (A t) d t
$$


Indeed,

$$
\begin{aligned}
A^{*} X+X A & =\int_{0}^{\infty} \frac{\partial}{\partial t}\left(\exp \left(A^{*} t\right) \exp (A t)\right) d t \\
& =-1 .
\end{aligned}
$$

Conversely, from (5.2) it follows, for an eigenvector $e$ of $A$ with an eigenvalue $\lambda$, that

$$
\begin{aligned}
(e, e) & =-(X A e, e)-(X e, A e) \\
& =-(\lambda+\bar{\lambda})(X e, e) .
\end{aligned}
$$

Hence $\Re \lambda<0$, as desired.

Proof of Theorem 5.1. If $A(x)$ is a smooth periodic function in $x$, then (5.3) shows that $X(x)$ is also a smooth periodic function. In particular, there are bounds independent of $x$, for

$$
0<C_{1} \leq X(x) \leq C_{2}
$$

in the sense of quadratic forms. Denoting the usual norm in $\mathbb{C}^{r}$ by $\|e\|=$ $\sqrt{(e, e)}$, we define a new norm

$$
\|e\|_{X}=\sqrt{(X e, e)}
$$

which is equivalent to the usual one. Then, inequalities (5.4) give a precise form of the equivalence relations

$$
C_{1}\|e\|^{2} \leq\|e\|_{X}^{2} \leq C_{2}\|e\|^{2} .
$$

For a solution $U(x, y, \tau)$, we consider the function

$$
\begin{aligned}
f(x) & =\|U e\|_{X}^{2} \\
& =\left(U^{*}(x, y, \tau) X(x) U(x, y, \tau) e, e\right) .
\end{aligned}
$$

Differentiating and using (5.2), we get

$$
\begin{aligned}
\frac{\partial f}{\partial x} & =\left(U^{*}\left((A \tau+B)^{*} X+X(A \tau+B)+X^{\prime}\right) U e, e\right) \\
& =-\tau(U e, U e)+\left(\left(B^{*} X+X B+X^{\prime}\right) U e, U e\right) .
\end{aligned}
$$

The matrix $B^{*} X+X B+X^{\prime}$ is Hermitian and, for $\tau$ large enough, we have

$$
-\frac{\tau}{2} \leq B^{*} X+X B+X^{\prime} \leq \frac{\tau}{2}
$$

in the sense of quadratic forms. By (5.5), the norm $\|U e\|^{2}$ may be replaced by $\|U e\|_{X}^{2}$, hence

$$
\frac{\partial f}{\partial x} \leq-d \tau f(x)
$$


with some positive constant $d$. Dividing by $f(x)$ and integrating from $y$ to $x$, with $x \geq y$, we obtain

$$
\log \frac{f(x)}{f(y)} \leq-d \tau(x-y)
$$

which means that

$$
\|U(x, y, \tau) e\|_{X(x)}^{2} \leq \exp (-d(x-y) \tau)\|e\|_{X(y)}^{2} .
$$

Since the norms $\|\cdot\|_{X(x)}$ are equivalent to any fixed norm $\|\cdot\|$, we come to (5.1), which completes the proof.

This theorem has some obvious modifications. For example, an estimate

$$
\left\|U(x, \tau) U^{-1}(y, \tau)\right\| \leq C \exp (-d(x-y) \tau)
$$

holds if $\tau \rightarrow-\infty$ and $x \leq y$. Next, we may replace a stable matrix $A=a_{-}$ by a matrix $A=a_{+}$with a spectrum in the right half-plane. In this case we have

$$
\left\|U(x, \tau) U^{-1}(y, \tau)\right\| \leq C \exp (d(x-y) \tau)
$$

for $\tau \rightarrow+\infty$ and $x \leq y$ or $\tau \rightarrow-\infty$ and $x \geq y$, with some $C, d>0$.

\section{Asymptotics of solutions.}

In this section we consider the general case of Equation (4.1) with a split matrix $A(x)$. So, we write it in the form

$$
\frac{\partial U}{\partial x}=(\Lambda(x, \tau)+B(x)) U
$$

where

$$
\begin{aligned}
\Lambda(x, \tau) & =\left(\begin{array}{cc}
\lambda_{+}(x, \tau) & 0 \\
0 & \lambda_{-}(x, \tau)
\end{array}\right) \\
& =\left(\begin{array}{cc}
a_{+}(x) \tau+b_{11}(x) & 0 \\
0 & a_{-}(x) \tau+b_{22}(x)
\end{array}\right)
\end{aligned}
$$

is a block-diagonal part and

$$
B(x)=\left(\begin{array}{cc}
0 & b_{12}(x) \\
b_{21}(x) & 0
\end{array}\right)
$$

is an antidiagonal part of the coefficients. We assume that both $a_{-}(x)$ and $-a_{+}(x)$ are stable matrices.

Let us look for a solution of (6.1) in the form (cf. (4.5) in [FT87, Ch. 1])

$$
U(x, \tau)=(1+W(x, \tau)) Z(x, \tau),
$$


where $Z$ is a block-diagonal matrix and $W$ is an antidiagonal matrix. Substituting (6.3) into (6.1) and separating diagonal and antidiagonal parts, we obtain

$$
\begin{aligned}
\frac{\partial W}{\partial x} Z+W \frac{\partial Z}{\partial x} & =\Lambda W Z+B Z \\
\frac{\partial Z}{\partial x} & =(\Lambda+B W) Z
\end{aligned}
$$

Eliminating $Z$, we arrive at a matrix Riccati equation for $W$

$$
\frac{\partial W}{\partial x}=\Lambda W-W \Lambda+B-W B W
$$

Were $W$ a solution of (6.5), the second equation in (6.4) would give us an equation for $Z$ with a block-diagonal coefficient $\Lambda+B W$.

To find $W$, we observe that Equation (6.5) is equivalent to two separate equations for $w_{12}$ and $w_{21}$,

$$
\begin{aligned}
& \frac{\partial w_{12}}{\partial x}=\lambda_{+} w_{12}-w_{12} \lambda_{-}+b_{12}-w_{12} b_{21} w_{12}, \\
& \frac{\partial w_{21}}{\partial x}=\lambda_{-} w_{21}-w_{21} \lambda_{+}+b_{21}-w_{21} b_{12} w_{21} .
\end{aligned}
$$

Assuming $\lambda_{ \pm}$to be of the form (6.2), let us consider $\tau$ positive and large enough. We will look for solutions to (6.6) and (6.7) on the closed interval $x \in[0,2 \pi]$ with initial conditions

$$
\begin{aligned}
w_{12}(2 \pi) & =0, \\
w_{21}(0) & =0 .
\end{aligned}
$$

Lemma 6.1. The solutions of (6.6), (6.8) and (6.7), (6.9) exist, for $\tau$ large enough, and satisfy the estimates

$$
\begin{aligned}
& \left\|w_{12}(x, \tau)\right\|=O\left(\frac{1}{\tau}\right) \\
& \left\|w_{21}(x, \tau)\right\|=O\left(\frac{1}{\tau}\right)
\end{aligned}
$$

uniformly in $x \in[0,2 \pi]$.

Proof. Let us consider the case of $w_{12}$, the reasoning for $w_{21}$ is similar. First we reduce (6.6), (6.8) to an equivalent integral equation. To this end, let us treat $f=b_{12}-w_{12} b_{21} w_{12}$ as a known function and apply the variation of constants to the equation

$$
w_{12}^{\prime}=\lambda_{+} w_{12}-w_{12} \lambda_{-}+f .
$$

In other words, we look for a solution of the form

$$
w_{12}(x)=U_{+}(x) V(x) U_{-}^{-1}(x)
$$


where $U_{ \pm}(x, \tau)$ are fundamental solutions to the Cauchy problems

$$
\begin{aligned}
\frac{\partial U_{ \pm}}{\partial x} & =\lambda_{ \pm} U_{ \pm}, \\
\left.U_{ \pm}\right|_{x=0} & =1 .
\end{aligned}
$$

Substituting, we obtain

$$
\frac{\partial V}{\partial x}=U_{+}^{-1} f U_{-}
$$

and taking into account (6.8),

$$
V(x)=-\int_{x}^{2 \pi} U_{+}^{-1}(y) f(y) U_{-}(y) d y .
$$

Now, returning to (6.11) and replacing $f(y)$, we come to the integral equation

$$
w_{12}(x)=-\int_{x}^{2 \pi} U_{+}(x) U_{+}^{-1}(y)\left(b_{12}(y)-w_{12}(y) b_{21}(y) w_{12}(y)\right) U_{-}(y) U_{-}^{-1}(x) d y .
$$

This equation may be solved by iterations. From Theorem 5.1 and what has been said at the end of Section 5 , we deduce that

$$
\begin{aligned}
& \left\|U_{+}(x) U_{+}^{-1}(y)\right\| \leq C \exp (d(x-y) \tau), \\
& \left\|U_{-}(y) U_{-}^{-1}(x)\right\| \leq C \exp (d(x-y) \tau)
\end{aligned}
$$

for $\tau \gg 1$ and $x \leq y$. In particular, these expressions are uniformly bounded for $\tau \gg 1$ and $0 \leq x \leq y \leq 2 \pi$. The initial iteration

$$
-\int_{x}^{2 \pi} U_{+}(x) U_{+}^{-1}(y) b_{12}(y) U_{-}(y) U_{-}^{-1}(x) d y
$$

may be estimated by means of (6.12), (6.13) as

$$
\begin{aligned}
C \int_{x}^{2 \pi} \exp (2 d(x-y) \tau) d y & \leq \frac{C}{2 d \tau} \\
& =O\left(\frac{1}{\tau}\right) .
\end{aligned}
$$

When combined with the boundedness of (6.12) and (6.13), this estimate implies the convergence of the iterations and the desired estimate (6.10).

Similarly, for $w_{21}$ we obtain an integral equation

$$
w_{21}(x)=\int_{0}^{x} U_{-}(x) U_{-}^{-1}(y)\left(b_{21}(y)-w_{21}(y) b_{12}(y) w_{21}(y)\right) U_{+}(y) U_{+}^{-1}(x) d y
$$

and then repeat the previous arguments. 
Turning to the block-diagonal part, we denote by $Z_{ \pm}(x, \tau)$ the entries of $Z$. More precisely, we take them as solutions of the Cauchy problems

$$
\begin{aligned}
\frac{\partial Z_{+}}{\partial x} & =\left(\lambda_{+}+b_{12} w_{21}\right) Z_{+}, \\
\left.Z_{+}\right|_{x=0} & =1
\end{aligned}
$$

and

$$
\begin{aligned}
\frac{\partial Z_{-}}{\partial x} & =\left(\lambda_{-}+b_{21} w_{12}\right) Z_{-}, \\
\left.Z_{-}\right|_{x=0} & =1 .
\end{aligned}
$$

The crucial property of the coefficients in (6.14) and (6.15) is that, for $\tau \gg 1$, the matrix

$$
\lambda_{-}+b_{21} w_{12}=\lambda_{-}+O\left(\frac{1}{\tau}\right)
$$

is stable and so is

$$
-\left(\lambda_{+}+b_{12} w_{21}\right) .
$$

In particular, this implies estimates (5.1), (5.6) for $Z_{-}$and (5.7) for $Z_{+}$.

We have thus constructed a solution of the form (6.3), with

$$
W(x, \tau)=O\left(\frac{1}{\tau}\right)
$$

uniformly in $x$. It does not satisfy the initial condition $U(0, \tau)=1$, but this drawback can be easily corrected. Indeed,

$$
\begin{aligned}
V(x, \tau) & =U(x, \tau) U^{-1}(0, \tau) \\
& =(1+W(x, \tau)) Z(x, \tau)(1+W(0, \tau))^{-1}
\end{aligned}
$$

is the desired solution. For the monodromy matrix, we obtain

$$
\begin{aligned}
M(\tau) & =V(2 \pi, \tau) \\
& =(1+W(2 \pi, \tau)) Z(2 \pi, \tau)(1+W(0, \tau))^{-1} \\
& =\left(1+O\left(\frac{1}{\tau}\right)\right)\left(\begin{array}{cc}
Z_{+}(2 \pi, \tau) & 0 \\
0 & Z_{-}(2 \pi, \tau)
\end{array}\right)\left(1+O\left(\frac{1}{\tau}\right)\right) .
\end{aligned}
$$

Finally, we apply (6.16) to compute the asymptotic expansion of the phase function

$$
\varphi(\tau)=\frac{1}{2} \log \operatorname{det}\left(M(\tau)+M^{-1}(\tau)-2\right)
$$


for $\Re \tau \rightarrow \pm \infty$ and $|\Im \tau| \leq C$. All the calculations will be performed modulo $\pi i$. From (6.16) it follows that

$$
\begin{aligned}
& M(\tau)+M^{-1}(\tau)-2=\left(1+O\left(\frac{1}{\tau}\right)\right)\left\{Z(2 \pi, \tau)\left(1+O\left(\frac{1}{\tau}\right)\right)\right. \\
& \left.+\left(1+O\left(\frac{1}{\tau}\right)\right) Z^{-1}(2 \pi, \tau)-2\left(1+O\left(\frac{1}{\tau}\right)\right)\right\}\left(1+O\left(\frac{1}{\tau}\right)\right)
\end{aligned}
$$

implying

$$
\begin{aligned}
\varphi(\tau) & =\frac{1}{2} \log \operatorname{det}\left\{Z(2 \pi, \tau)\left(1+O\left(\frac{1}{\tau}\right)\right)\right. \\
& \left.+\left(1+O\left(\frac{1}{\tau}\right)\right) Z^{-1}(2 \pi, \tau)-2\left(1+O\left(\frac{1}{\tau}\right)\right)\right\}+O\left(\frac{1}{\tau}\right) .
\end{aligned}
$$

A straightforward computation shows that the expression in curly brackets transforms further to

$$
\begin{aligned}
& \left(\begin{array}{cc}
Z_{+}(2 \pi, \tau) & 0 \\
0 & 1
\end{array}\right)\left\{\left(\begin{array}{cc}
1 & 0 \\
0 & Z_{-}(2 \pi, \tau)
\end{array}\right)\left(1+O\left(\frac{1}{\tau}\right)\right)\left(\begin{array}{cc}
1 & 0 \\
0 & Z_{-}(2 \pi, \tau)
\end{array}\right)\right. \\
& +\left(\begin{array}{cc}
Z_{+}^{-1}(2 \pi, \tau) & 0 \\
0 & 1
\end{array}\right)\left(1+O\left(\frac{1}{\tau}\right)\right)\left(\begin{array}{cc}
Z_{+}^{-1}(2 \pi, \tau) & 0 \\
0 & 1
\end{array}\right) \\
& \left.-2\left(\begin{array}{cc}
Z_{+}^{-1}(2 \pi, \tau) & 0 \\
0 & 1
\end{array}\right)\left(1+O\left(\frac{1}{\tau}\right)\right)\left(\begin{array}{cc}
1 & 0 \\
0 & Z_{-}(2 \pi, \tau)
\end{array}\right)\right\}\left(\begin{array}{cc}
1 & 0 \\
0 & Z_{-}^{-1}(2 \pi, \tau)
\end{array}\right) .
\end{aligned}
$$

Now, $Z_{+}^{-1}(2 \pi, \tau)$ and $Z_{-}(2 \pi, \tau)$ decay exponentially for $\Re \tau \rightarrow+\infty$. Indeed, applying (5.1) for $x=2 \pi$ and $y=0$, we get

$$
\begin{aligned}
\left\|Z_{-}(2 \pi, \tau)\right\| & \leq C \exp (-2 \pi d \tau) \\
& =O\left(\frac{1}{\tau}\right) ;
\end{aligned}
$$

the same is true for $Z_{+}^{-1}(2 \pi, \tau)$, as may be seen from (5.7) for $x=0$ and $y=2 \pi$. Hence the previous expression can be rewritten as

$$
\left(\begin{array}{cc}
Z_{+}(2 \pi, \tau) & 0 \\
0 & 1
\end{array}\right)\left(1+O\left(\frac{1}{\tau}\right)\right)\left(\begin{array}{cc}
1 & 0 \\
0 & Z_{-}^{-1}(2 \pi, \tau)
\end{array}\right)
$$

so that

$$
\varphi(\tau)=\frac{1}{2} \log \operatorname{det} Z_{+}(2 \pi, \tau)-\frac{1}{2} \log \operatorname{det} Z_{-}(2 \pi, \tau)+O\left(\frac{1}{\tau}\right) .
$$


Finally, using the Liouville formula for $\operatorname{det} Z_{+}$and $\operatorname{det} Z_{-}$, we arrive at $(6.17)$

$$
\begin{aligned}
\varphi(\tau) & =\frac{1}{2} \int_{0}^{2 \pi} \operatorname{tr}\left(\lambda_{+}+b_{12} w_{21}\right) d x-\frac{1}{2} \int_{0}^{2 \pi} \operatorname{tr}\left(\lambda_{-}+b_{21} w_{12}\right) d x+O\left(\frac{1}{\tau}\right) \\
& =\frac{1}{2} \int_{0}^{2 \pi}\left(\operatorname{tr}\left(a_{+}(x) \tau+b_{11}(x)\right)-\operatorname{tr}\left(a_{-}(x) \tau+b_{22}(x)\right)\right) d x+O\left(\frac{1}{\tau}\right) .
\end{aligned}
$$

Similarly, an asymptotic formula for $\varphi(\tau)$ may be obtained as $\Re \tau \rightarrow-\infty$ and $|\Im \tau| \leq C$. The result will be given by (6.17) with the opposite sign. We summarize these results as follows.

Theorem 6.2. Let $\Re \tau \rightarrow \pm \infty$ and $|\Im \tau| \leq C$. Then the following asymptotic formulas hold:

$$
\begin{aligned}
\varphi(\tau)= & \pm \frac{1}{2} \int_{0}^{2 \pi}\left(\operatorname{tr}\left(a_{+}(x) \tau+b_{11}(x)\right)-\operatorname{tr}\left(a_{-}(x) \tau+b_{22}(x)\right)\right) d x+\pi i N_{ \pm} \\
& +O\left(\frac{1}{\tau}\right) .
\end{aligned}
$$

The integers $N_{ \pm}$remain undetermined. We may fix one of them, then the other will depend on the path to be used for analytic extension.

Corollary 6.3. The variation $\Delta_{\Gamma, \tau_{0}} \varphi(\tau)$ is an integer multiple of $\pi i$.

Proof. Using (6.18) we write

$$
\begin{aligned}
& \Delta_{\Gamma, \tau_{0}} \varphi(\tau)=\pi i\left(N_{+}-N_{-}\right) \\
& \quad+\frac{1}{2} \int_{0}^{2 \pi}\left(\operatorname{tr}\left(a_{+}(x)\left(\tau_{0}+T\right)+b_{11}(x)\right)-\operatorname{tr}\left(a_{-}(x)\left(\tau_{0}+T\right)+b_{22}(x)\right)\right) d x \\
& \quad+\frac{1}{2} \int_{0}^{2 \pi}\left(\operatorname{tr}\left(a_{+}(x)\left(\tau_{0}-T\right)+b_{11}(x)\right)-\operatorname{tr}\left(a_{-}(x)\left(\tau_{0}-T\right)+b_{22}(x)\right)\right) d x .
\end{aligned}
$$

The two integral terms give

$$
\int_{0}^{2 \pi}\left(\operatorname{tr}\left(a_{+}(x) \tau_{0}+b_{11}(x)\right)-\operatorname{tr}\left(a_{-}(x) \tau_{0}+b_{22}(x)\right)\right) d x
$$

which is zero in virtue of Lemma 4.1 .

\section{References}

[APS75] M.F. Atiyah, V.K. Patodi and I.M. Singer, Spectral asymmetry and Riemannian geometry, I, Math. Proc. Camb. Phil. Soc., 77 (1975), 43-69.

[BFK91] D. Burghelea, L. Friedlander and T. Kappeler, On the determinant of elliptic differential and finite difference operators in vector bundles over $\mathbb{S}^{1}$, Commun. Math. Phys., 138 (1991), 1-18. 
[FT87] L.D. Faddeev and L.A. Takhtajan, Hamiltonian Methods in the Theory of Solitons, Springer-Verlag, Berlin et al., 1987.

[FST97] B.V. Fedosov, B.-W. Schulze and N.N. Tarkhanov, The Index of Elliptic Operators on Manifolds with Conical Points, Preprint 97/24, Univ. Potsdam, Potsdam, August 1997.

[FST97] _ On the Index Formula for Singular Surfaces, Preprint 97/31, Univ. Potsdam, Potsdam, November 1997.

[Gan86] F.R. Gantmacher, The Theory of Matrices, Springer-Verlag, Berlin et al., 1986.

[GS83] P.B. Gilkey and L. Smith, The twisted index problem for manifolds with boundary, J. Differential Geom., 18 (1983), 393-444.

[Mel93] R.B. Melrose, The Atiyah-Patodi-Singer Index Theorem, A K Peters, Wellesley, Mass., 1993.

[Mel95] _ , The eta invariant and families of pseudodifferential operators, Math. Res. Letters, 2(5) (1995), 541-561.

[SSS97] B.-W. Schulze, B. Sternin and V. Shatalov, On the Index of Differential Operators on Manifolds with Conical Singularities, Preprint 97/10, Univ. Potsdam, Potsdam, May 1997.

Received March 1, 1998. The third author was supported by the Max-Planck Gesellschaft.

UNIVERSiTÄT POTSDAM

14415 PotSDAM

Germany

UNIVERSITÄT PotSDAM

14415 PotSDAM

Germany

UnIVERSität Potsdam

14415 Potsdam

Germany

E-mail address: tarkhan@mpg-ana.uni-potsdam.de 